Philip Thomas

\title{
The length and severity of the coronavirus recession estimated from the dynamics of relaxing lockdown
}

\begin{abstract}
The J-value is used to cast light on the policy of lockdown and its relaxation by linking findings from epidemiological and economic analysis. A new, two-cohort model is applied to the coronavirus epidemic to allow for different sections of the population exhibiting different responses to the same disease. Optimal parameter sets are generated by adjusting the model to fit the data on new daily cases in England up to 10 April 2020. While several sets of parameter values give an equally good match to the data, a common feature is that disease transients for the controlled second wave of the disease are long drawn out, with tails resulting from an easing of restrictions in May 2020 lasting to the end of 2021 or later. A further feature in common is the lack of population ("herd") immunity at the end of the second wave, which leaves the population of England, and the UK by extension, open to further significant waves of the infection. While considerable uncertainties remain with the epidemiology, there is no doubt that the positive feedback nature of the COVID-19 epidemic will make controlling the outbreak present in May 2020 a very difficult task, especially if the main regulating tool selected is the coordinated behaviour of 67 million people. The likely economic effect of the years-long process to move out of lockdown sufficiently slowly so as not to cause excessive strain on the health services is analysed. In the extended base case, annual GDP will fall by $23.5 \%$ in 2020 and is not likely to recover to pre-lockdown levels until 2024. Applying the J-value-derived Bristol curve of population-average life-expectancy versus GDP suggests that the strategy of restriction followed by gradual relaxation is likely to result in a net cost, in terms of average human lives lost, that will be comparable with the UK's sacrifice over the six years of World War Two. A policy of lockdown followed by gradual relaxation is likely to do much more harm to the nation's health than good.
\end{abstract}

Keywords: coronavirus, COVID-19, economic challenge, J-value, lockdown, lockdown exit strategy, multicohort epidemic model

Nanotechnology Perceptions 16 (2020) 100-129

doi: 10.4024/N07TH20A.ntp.16.02 